\title{
ARTICLE Metabolism, pharmacokinetics, and hepatic disposition of xanthones and saponins on Zhimu treatments for exploratively interpreting the discrepancy between the herbal safety and timosaponin A3-induced hepatotoxicity
}

\author{
Yang Xie ${ }^{1,2}$, Xu Zhou ${ }^{1,3}$, Hu Pei ${ }^{1,3}$, Ming-cang Chen ${ }^{1,3}$, Zhao-lin Sun ${ }^{1,3}$, Ya-ru Xue ${ }^{1,3}$, Xiao-ting Tian ${ }^{1,3}$ and Cheng-gang Huang ${ }^{1,3}$
}

Timosaponin A3, a saponin in Zhimu, elicited hepatotoxicity via oxidative stress. However, the clinical medication of Zhimu has been historically regarded as safe, probably associated with the antioxidants it contains. However, the related information on the in vivo levels of timosaponin A3 and antioxidants remained unclear on Zhimu treatments. Therefore, a combination of the in vitro metabolism, including microbiota-mediated and liver-mediated metabolism, and in vivo pharmacokinetics and hepatic disposition, was conducted for three xanthones (neomangiferin, mangiferin, and norathyriol) and three saponins (timosaponin B2, timosaponin B3, and timosaponin A3) on Zhimu treatments. Consequently, following oral administration of Zhimu decoction to rats, those saponins and xanthones were all observed in the plasma with severe liver first-pass effect, where mangiferin was of the maximum exposure. Despite the ignorable content in the herb, timosaponin A3 elicited sizable hepatic exposure as the microbiota-mediated metabolite of saponins in Zhimu. The similar phenomenon also occurred to norathyriol, the microbiota-mediated metabolite of xanthones. However, the major prototypes in Zhimu were of limited hepatic exposure. We deduced the hepatic collection of norathyriol, maximum circulating levels of mangiferin, and timosaponin B2 and mangiferin interaction may directly or indirectly contribute to the whole anti-oxidation of Zhimu, and then resisted the timosaponin A3-induced hepatotoxicity. Thus, our study exploratively interpreted the discrepancy between herbal safety and timosaponin A3-induced hepatotoxicity. However, given the considerable levels and slow eliminated rate of timosaponin A3 in the liver, more attention should be paid to the safety on the continuous clinical medication of Zhimu in the future.

Keywords: Zhimu; timosaponin A3; mangiferin; pharmacokinetics; metabolism; hepatotoxicity; antioxidant

Acta Pharmacologica Sinica (2018) 39:1923-1934; https://doi.org/10.1038/s41401-018-0012-z

\section{INTRODUCTION}

With the increasing consumption of herb as medicine or dietary supplements around the world, the safety of herb, especially for the herbal hepatotoxicity, has become a major public concern [1]. According to the estimation by the US Drug-Induced Liver Injury Network, herbal consumption-induced liver injury accounted for $16 \%$ of all cases reported [2]. More importantly, this proportion increased from $7 \%$ in 2004-2005 to $20 \%$ in 2013-2014 [2]. Nowadays, there has been a hot and sharp controversy on the relationship between the liver cancer and aristolochic acids (AA), a metabolism-based toxic ingredient widely distributed in the genera Aristolochia and Asarum, which trigged by the report that $78 \%$ of hepatocellular carcinomas samples showed distinctive mutational signature of AA among 98 patients in Taiwan, China [3]. Once observing the toxic ingredients in the herb, or the toxic metabolites in vivo, it is timely indispensable and essential to clarify the exposure of those toxic substances in vivo, especially paying more attention to the organs associated with toxicity. It is worth noting, however, that the herbs containing toxic ingredients or metabolites do not equal to their unsafe usage in clinic. Given the inherent nature of herb containing various ingredients, the possible drug-drug interaction (DDI) at pharmacokinetic or pharmacodynamic levels probably cause the toxicity enhancement or alleviation on herb treatments [4].

Zhimu (Rhizoma anemarrhenae), botanically from the dried rhizome of Anemarrhena asphodeloides Bge, has been one of the most frequently used herb medicines in China for centuries with various activities, such as antipyretic, anti-inflammatory, antioxidation, anti-depressant, and anti-diabetes [5-9]. According to the isolated compounds and pharmacological screenings, the active ingredients in Zhimu were mainly xanthones, represented by neomangiferin and mangiferin (Fig. 1a), and saponins, represented by timosaponin B2 and timosaponin B3 (Fig. 1b), with lots of activities [9]. Nowadays, the anti-diabetic activities of mangiferin, as well as the anti-depressant and anti-dementia effect of timosaponin B2, attracted extensive attention [10-12]. Our team has been focused on those active compounds in Zhimu for many years, and a great body of investigations have been

\footnotetext{
${ }^{1}$ Shanghai Institute of Materia Medica, Chinese Academy of Sciences, Shanghai 201203, China; ${ }^{2}$ Pharmacy Academy, Harbin University of Commerce, Harbin 150076, China and ${ }^{3}$ University of Chinese Academy of Sciences, Beijing 100049, China

Correspondence: Xiao-ting Tian (zhghydxtxt@163.com) or Cheng-gang Huang (cghsimm@126.com)
}

Received: 11 December 2017 Accepted: 31 January 2018

Published online: 23 May 2018 


\section{a Xanthones}

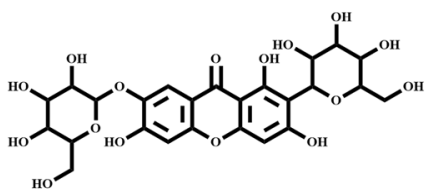

Neomangiferin<smiles>CCC1CC(c2c(C)cc3oc4cc(C)c(C)cc4c(=O)c3c2C)C(C)C(C)C1C</smiles>

Mangiferin

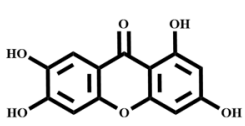

Norathyriol

\section{b Saponins}

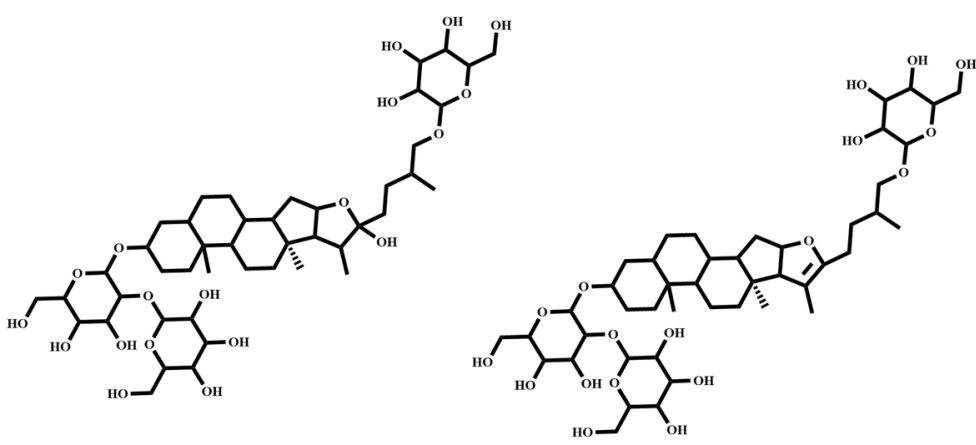

Timosaponin B2

Timosaponin B3

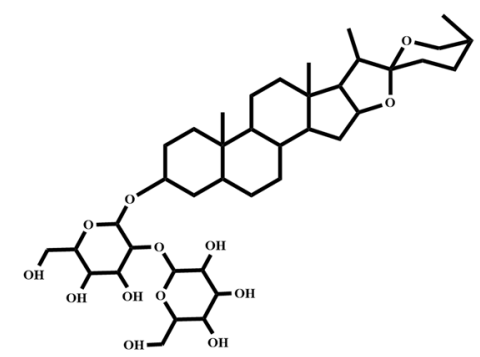

Timosaponin A3

Fig. 1 Chemical structures of three xanthones (a) and three saponins (b) in Zhimu

performed, including the metabolic patterns of mangiferin $[13,14]$, timosaponin B2 $[15,16]$, timosaponin B3 [17] and timosaponin A3 [18], the pharmacokinetic profiles of mangiferin $[19,20]$, the hepatobiliary disposition of timosaponin B2 [21], and the DDI between timosaponin B2 and mangiferin [20]. Those provided much scientific data for understanding the effective material base of Zhimu.

Although there were no related reports regarding the toxicity during the historically clinical medication of Zhimu [9], we previously discovered obvious timosaponin A3-induced hepatotoxicity in rats, a steroid saponin isolated from Zhimu, following oral administration of timosaponin A3 for consecutive 14 days (100 mg/kg per day) [22]. We also confirmed this hepatotoxicity was highly associated with timosaponin A3-induced oxidative stress, and the presence of antioxidants can powerfully fight against the timosaponin A3-induced hepatotoxicity in rat hepatocytes [22]. As published, xanthones were good scavengers for various oxidizing species, and the antioxidant capacity of xanthones was profoundly correlated with the number and position of hydroxyl and catechol groups [23]. Correspondingly, we also previously confirmed mangiferin, a xanthone with strong antioxidant ability in Zhimu [24, 25], can powerfully attenuate the oxidative stress induced by timosaponin A3 in hepatocytes [22]. Nevertheless, such an in vitro results were far less than comprehensively interpreting the safe clinical medication of Zhimu for centuries, particularly in the absence of the in vivo levels of antioxidants and timosaponin A3 on Zhimu treatments. Moreover, according to our previous study, timosaponin A3 was detected as a major metabolite after oral administration of timosaponin B2 or timosaponin B3 in rats $[15,17]$, two saponins with the high content in Zhimu, suggesting a high probability of substantial biodistribution of timosaponin A3 on Zhimu treatments. Therefore, in light of the complicated transformation among saponins, and the confrontation relationship on the hepatotoxicity between timosaponin $\mathrm{A} 3$ and xanthones, it was imperative to make it clear for the in vivo dynamic disposition of those xanthones and saponins, especially for the hepatic disposition. However, up to date, in spite of several studies only concerning the circulating levels of those ingredients on Zhimu treatments [26-28], the major issues regarding the safety of Zhimu, such as the liver distribution of timosaponin A3, the formation pathway of timosaponin $A 3$, and the exposure relationship between timosaponin $A 3$ and the prototypes or metabolites with antioxidant activity, and so on, remained largely unclear.

Therefore, aimed at clarifying the metabolic patterns, the in vitro tools, rat primary hepatocytes and enterobacteria, were employed for evaluating the liver-mediated and microbiotamediated metabolism of xanthones and saponins in Zhimu, respectively. On the basis of those results, after oral administration of Zhimu decoction in rats, the determination of the prototypes and metabolites with the skeleton structure of saponin and xanthone was systemically conducted in the plasma before and after hepatic disposition, namely portal vein plasma and systemic plasma, as well as liver. Serving as the technical foundation, a sensitive and accurate HPLC-MS/MS method was developed and validated for the simultaneous determination of three xanthones, neomangiferin, mangiferin, and norathyriol, and three saponins, timosaponin B2, timosaponin B3, and timosaponin A3, in the plasma and liver. Hence, via the systemic evaluation on the pharmacokinetics, metabolism, and hepatic disposition of ingredients in Zhimu, this study was aimed at potentially understanding the discrepancy between the historically clinical safety of Zhimu and timosaponin A3-induced hepatotoxicity.

\section{MATERIALS AND METHODS}

Materials

Zhimu was purchased from Anhui Shuzhong traditional Chinese Medicine Co., Ltd (Shanghai, China), and were identified as dried rhizome of Anemarrhena asphodeloides Bge by Dr. Zhixiong Li. Norathyriol (purity >98\%) was isolated from rat urine postmangiferin dosing in our laboratory [13]. Calycosin-7-O- $\beta-D-$ glucopyranoside $(\mathrm{C} 7 \mathrm{G})$, mangiferin, neomangiferin, timosaponin B2, timosaponin B3, timosaponin A3, and tolbutamide (internal 
standard, IS) were bought from the National Institute for Control of Pharmaceutical and Biological Products Co., Ltd (Beijing, China). Acetonitrile and formic acid of HPLC grade were obtained from Fisher Scientific Co., Ltd (Tustin, CA, USA). All other analyticalgrade reagents were provided by Sinopharm Chemical Reagent Co., Ltd (Shanghai, China) or Sigma-Aldrich Co., Ltd (St. Louis, MO, USA). Triple-deionized water was prepared using a Milli-Q System (Millipore, Billerica, MA, USA). Anaerobic bags were bought from AnaeroPouch $^{\text {TM }}$-Anaero, Mitsubishi Gas Chemical Co., Ltd (Tokyo, Japan), and anaerobic culture solution was purchased from Qingdao Hope Biotechnology Co., Ltd (Qingdao, China).

\section{Instrumentation}

The determination of three xanthones and three saponins in different biological samples was performed by an Agilent 6460 triple quadrupole mass spectrometer with Dual Agilent Jet Stream Electrospray lonization (Agilent Technologies, Palo Alto, CA, USA), combining with an Agilent 1260 Series Liquid Chromatography System (Agilent Technologies, Palo Alto, CA, USA). The MS parameters were optimized as follows: gas temperature, $350^{\circ} \mathrm{C}$; gas flow rate, $12 \mathrm{~L} / \mathrm{min}$; nebulizer, $45 \mathrm{psi}$; capillary, $3500 \mathrm{~V}$; nozzle voltage, $1000 \mathrm{~V}$; sheath gas temperature, $400^{\circ} \mathrm{C}$; sheath gas flow rate, $8 \mathrm{~L} / \mathrm{min}$. All quantifications of compounds were monitored in the negative multiple reaction monitoring (MRM) mode, and the relevant optimized parameters were shown in Table 1. The chromatographic separation was carried out using an ACE Super C18 $(100 \mathrm{~mm} \times 2.1 \mathrm{~mm}, 3 \mu \mathrm{m}$, Advanced Chromatography Technologies Limited, Aberdeen, Scotland) at the column temperature of $40^{\circ} \mathrm{C}$. A binary gradient consisting of solvent $A$ (water containing $0.1 \%$ formic acid) and solvent $B$ (acetonitrile containing $0.1 \%$ formic acid) was employed as follows: 0-3 min, 92\% A; 3.5-4 $\min , 88 \%-60 \%$ A; 5.5-6 $\min , 60 \%-55 \%$ A; 6.6-7 min, 55\%-5\% A; $11-11.01 \mathrm{~min}, 5 \%-92 \% \mathrm{~A} ; 11.01-13.5 \mathrm{~min}, 92 \% \mathrm{~A}$. The flow rate remained at $0.35 \mathrm{~mL} / \mathrm{min}$ at the time range of $0-6.6 \mathrm{~min}$, and it kept at $0.45 \mathrm{~mL} / \mathrm{min}$ at the time range of 7-13.5 $\mathrm{min}$. The system operation and data analysis were performed using Masshunter Workstation software (Agilent Technologies, Palo Alto, CA, USA).

\section{Preparation of the Zhimu decoction}

After soaking for $30 \mathrm{~min}$, the Zhimu pieces $(560 \mathrm{~g})$ were extracted by direct heating with $5.6 \mathrm{~L}$ of water for $2 \mathrm{~h}$, and after filtration, the residue was extracted by direct heating with $4.48 \mathrm{~L}$ of water for an additional two hours. After filtration, the combined supernatant was concentrated to $800 \mathrm{~mL}(0.7 \mathrm{~g} / \mathrm{mL})$ for analysis and oral administration to rats.

In vitro: microbiota-mediated metabolism

Anaerobic culture was performed as our previous literature described [14, 19, 29]. In brief, fresh intestinal contents obtained from Wistar rats were homogenized with saline solution $(0.9 \%)$ in the ratio of $1 \mathrm{~g}: 5 \mathrm{~mL}$, and then filtrated with gauze immediately. Zhimu decoction and the above filtrate contacting enterobacteria

Table 1. The optimized parameters of six ingredients in Zhimu and IS in the negative MRM mode of HPLC-QQQ-MS/MS

\begin{tabular}{llll}
\hline Analytes & Transition $(\mathrm{m} / \mathrm{z})$ & $\begin{array}{l}\text { Fragmentor } \\
\text { voltage }(\mathrm{v})\end{array}$ & $\begin{array}{l}\text { Collision } \\
\text { energy }(\mathrm{v})\end{array}$ \\
\hline Neomangiferin & $582.9 \rightarrow 330.9$ & 209 & 39 \\
Mangiferin & $420.9 \rightarrow 330.9$ & 149 & 22 \\
Norathyriol & $258.9 \rightarrow 150.8$ & 138 & 42 \\
Timosaponin B2 & $919.2 \rightarrow 756.9$ & 285 & 49 \\
Timosaponin B3 & $901.3 \rightarrow 739.1$ & 310 & 46 \\
Timosaponin A3 & $739.1 \rightarrow 577.2$ & 255 & 38 \\
Tolbutamide (IS) & $269 \rightarrow 170$ & 100 & 32 \\
\hline
\end{tabular}

were added to above intestinal flora anaerobic cultural solution to generate the concentration of Zhimu at $0.7 \mathrm{mg} / \mathrm{mL}$, and then cultured in the anaerobic incubation bags at $37^{\circ} \mathrm{C}(n=3)$. C7G (20 $\mu \mathrm{g} / \mathrm{mL}$ ) was used as a positive control. After incubation for 0,8 , and $24 \mathrm{~h}$, the incubated solution $(200 \mu \mathrm{L})$ was terminated by adding ice-cold methanol $(600 \mu \mathrm{L})$ immediately. The enterobacteria samples were diluted with methanol by a factor of 10 , and then extracted by acetonitrile containing 3\% acetic acid precipitation and prepared for analysis by HPLC-MS/MS.

In vitro: liver-mediated metabolism

The fresh hepatocytes were isolated from male Wistar rats by a previously described two-step perfusion [21, 30]. Briefly, the hepatocytes were incubated in six-well plates at a density of $0.5 \times$ $10^{6}$ per well followed by immediate addition of Zhimu decoction $(0.7$ $\mathrm{mg} / \mathrm{mL})$, and then incubated in an orbital shaker at $37^{\circ} \mathrm{C}$ for $3 \mathrm{~h}(n=$ 3). Testosterone $(10 \mu \mathrm{mol} / \mathrm{L})$ was used as positive control. Cell suspensions $(200 \mu \mathrm{L})$ were obtained at $0,1.5$, and $3 \mathrm{~h}$, and then icecold methanol $(600 \mu \mathrm{L})$ was immediately added to terminate the potential reactions. The hepatocyte samples were lysed after three successive freezes $\left(-80^{\circ} \mathrm{C}\right)$-thaw $\left(37^{\circ} \mathrm{C}\right)$ cycles, diluted with methanol by a factor of 10 , extracted by acetonitrile containing $3 \%$ acetic acid precipitation, and prepared for analysis by HPLC-MS/MS.

In vivo study

Male Wistar rats $(220 \pm 20 \mathrm{~g})$ were provided by Shanghai SLAC Laboratory Animal Co., Ltd (Shanghai, China). They were acclimated for seven days before use. The animals were housed under a controlled temperature of $22 \pm 2{ }^{\circ} \mathrm{C}$, relative humidity of $50 \% \pm 10 \%$, and $12 \mathrm{~h}$ light/dark cycle in the air-conditioned animal quarters. The rats were given ad libitum access to standard diet and water. The experiments were carried out in accordance with the guidelines by the Institutional Animal Care and Use Committee of Shanghai Institute of Materia Medica, China Academic Science (Shanghai, China).

Fifty rats were fasted overnight with the free access to water before use. After oral administration of Zhimu decoction $(7 \mathrm{~g} / \mathrm{kg}$, $0.7 \mathrm{~g} / \mathrm{mL})$, the rats were anaesthetized with urethane $(1.4 \mathrm{~g} / \mathrm{kg})$ at $0.167,0.5,1,2,4,6,8,11,24,48 \mathrm{~h}$ post-dose ( $n=5$ for each timepoint). Hepatic portal vein blood $(2 \mathrm{~mL})$ was collected firstly by syringe, and the systemic blood was obtained via drawing plasma from the abdominal aorta $(6-8 \mathrm{~mL})$. Finally, the right liver lobe was excised by tweezers. The blood samples were centrifuged at $9500 \times g$ for $5 \mathrm{~min}$ to obtain the supernatants; the liver samples were washed with saline for three times; all the above samples were stored at $-80^{\circ} \mathrm{C}$ until analysis.

\section{Sample preparation}

Standard stock solutions of neomangiferin, mangiferin, norathyriol, timosaponin B2, timosaponin B3, and timosaponin A3 were separately prepared in acetonitrile at $500 \mu \mathrm{g} / \mathrm{mL}$. The stock solutions were serially diluted with acetonitrile to prepare the working solutions. The calibration standards were prepared by spiking the working solutions to the biological samples to yield the final concentrations at $3,10,25,50,100,250,500$, and 1000 $\mathrm{ng} / \mathrm{mL}$ for each analyst, along with tolbutamide (IS) at $5 \mathrm{ng} / \mathrm{mL}$. The low, middle, and high concentration levels of quality control (QC) samples containing each analyte $(8,80$, and $800 \mathrm{ng} / \mathrm{mL})$ and IS $(5 \mathrm{ng} / \mathrm{mL})$ were prepared in the same manner.

The liver samples $(1 \mathrm{~g})$ were homogenized with three volumes $(3 \mathrm{~mL})$ of saline. The liver homogenates $(100 \mu \mathrm{L})$ or plasma samples $(100 \mu \mathrm{L})$ were precipitated through protein precipitation using acetonitrile containing $3 \%$ acetic acid $(300 \mu \mathrm{L})$. After mixed for $5 \mathrm{~min}$ and centrifuged at $13,680 \times \mathrm{g}$ for $10 \mathrm{~min}$, the supernatants $(250 \mu \mathrm{L})$ were separated and evaporated to dryness under vacuum at $40^{\circ} \mathrm{C}$. The residues were reconstituted with solvents $(100 \mu \mathrm{L})$, a combination of water, acetonitrile and acetic acid in the ratio of 92:8:0.1. After vortexed for $5 \mathrm{~min}$ and centrifuged at $13,680 \times \mathrm{g}$ for 
10 min, the supernatant $(70 \mu \mathrm{L})$ was obtained and prepared for the analysis by HPLC-MS/MS.

\section{Method validation}

The full validations, including of selectivity, linearity, accuracy, precision, matrix effect, extraction recovery, and stability, were performed in the plasma and liver matrix.

Selectivity was assessed by analyzing six different sources of blank biological samples, blank samples spiked with analytes and postdose biological samples from rats to determine potential interferences for the analytes and IS. Standard calibration curves were generated by plotting the peak area ratios of the analytes to the IS versus the concentrations of the analytes using a $1 / C$ - or $1 / C^{2}$ weighted linear least-squares regression model. The concentrations of ingredients in the biological samples were all calculated based on the regression parameters derived from the standard calibration curves prepared on the same day. The requirements for the lowest limit of quantification (LLOQ) were the lowest concentration with a signal-to-noise ratio $>10$, an accuracy within $100 \% \pm 20 \%$, and a precision (relative standard deviation, RSD) $<20 \%$.

The accuracy and precision of the method were evaluated by analyzing QC samples $(n=5)$ at three levels on one day (intra-day) and three successive days (inter-day). The stability was assessed by analyzing QC samples $(n=5)$ at three levels under different conditions, including after storage at an ambient temperature for 4 $\mathrm{h}$ (short-term stability), after storage at $-80^{\circ} \mathrm{C}$ for 2 weeks (long-term stability), after three successive freeze-thaw cycles (free-thaw stability) and after storage at room temperature for $12 \mathrm{~h}$ (post-preparative stability). Variation in the precision (RSD) and accuracy should be no more than $15 \%$ for the precision and stability validation.

Extraction recovery was evaluated by comparing the mean peak areas of the analytes and IS after the above-mentioned routine processing with those of the post-extraction spiked samples at the same concentration. The matrix effect was determined by comparing the mean peak areas of the analytes and IS of the post-extraction spiked samples with those of the standard
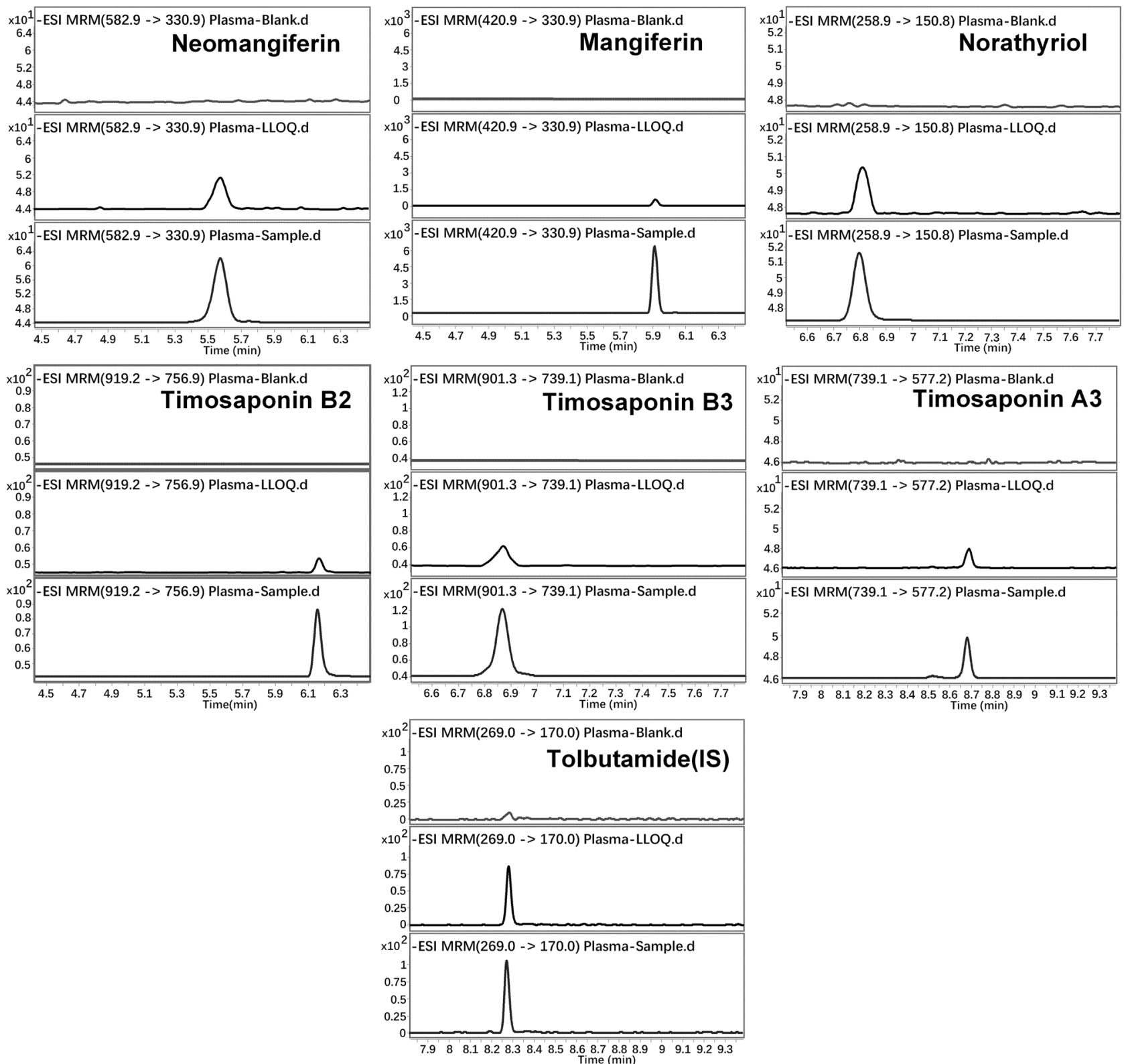

Fig. 2 Representative MRM chromatograms of six ingredients in Zhimu and IS in the blank plasma, blank plasma spiked with the analysts (3 $\mathrm{ng} / \mathrm{mL}, \mathrm{LLOQ})$ and IS $(5 \mathrm{ng} / \mathrm{mL})$, and plasma samples after oral administration of Zhimu decoction $(7 \mathrm{~g} / \mathrm{kg})$ at $2 \mathrm{~h}$ 


\begin{tabular}{|c|c|c|c|c|c|c|c|}
\hline Compound & Spiked (ng/mL) & Measured (ng/mL) & Accuracy (\%) & Precision (RSD\%) & Measured $(\mathrm{ng} / \mathrm{mL})$ & Accuracy (\%) & Precision (RSD\%) \\
\hline \multirow[t]{3}{*}{ Neomangiferin } & 8 & 8.2 & 102.5 & 2.9 & 7.8 & 97.4 & 4 \\
\hline & 80 & 85.9 & 107.4 & 6.7 & 90.2 & 112.7 & 2 \\
\hline & 800 & 792.9 & 99.1 & 5.3 & 839.9 & 105 & 5.2 \\
\hline & 80 & 85.5 & 106.9 & 5 & 83.5 & 104.4 & 4.8 \\
\hline & 800 & 810.1 & 101.3 & 4.4 & 806.4 & 100.8 & 4.4 \\
\hline \multirow[t]{3}{*}{ Norathyriol } & 8 & 7.4 & 92.5 & 4.9 & 7.9 & 98.4 & 4.9 \\
\hline & 80 & 84.1 & 105.1 & 5.9 & 70.4 & 88 & 9.5 \\
\hline & 800 & 827.1 & 103.4 & 10.1 & 765.5 & 95.7 & 7.4 \\
\hline \multirow{2}{*}{ Timosaponin B3 } & 80 & 77.4 & 96.8 & 6.2 & 79.1 & 98.9 & 4.2 \\
\hline & 800 & 803.9 & 100.5 & 9.3 & 780.4 & 97.6 & 10 \\
\hline \multirow[t]{3}{*}{ Timosaponin A3 } & 8 & 8 & 100 & 6.9 & 8.2 & 101.9 & 3.2 \\
\hline & 80 & 83.1 & 103.9 & 2.2 & 77.1 & 96.4 & 7.5 \\
\hline & 800 & 820.5 & 102.6 & 9.1 & 840.5 & 105.1 & 7.6 \\
\hline
\end{tabular}

Table 3. Extraction recovery and matrix effect of six ingredients in Zhimu and IS in the plasma ( $n=6)$

\begin{tabular}{|c|c|c|c|c|c|c|}
\hline Compound & \multicolumn{3}{|c|}{ Mean extraction recovery (\%) } & \multicolumn{3}{|c|}{ Matrix effect (\%) } \\
\hline Neomangiferin & $70.1 \pm 1.2$ & $70.6 \pm 1.7$ & $76.3 \pm 1.5$ & $110.3 \pm 5.3$ & $105 \pm 1.1$ & $111.1 \pm 4.5$ \\
\hline Mangiferin & $56.9 \pm 5.2$ & $53.9 \pm 2.8$ & $60.1 \pm 2$ & $110.2 \pm 6.6$ & $101.2 \pm 2.4$ & $105.1 \pm 3.5$ \\
\hline Norathyriol & $50 \pm 3.3$ & $47.9 \pm 1.5$ & $51.7 \pm 1.1$ & $111.3 \pm 4.3$ & $108.5 \pm 2$ & $101.4 \pm 4.2$ \\
\hline Timosaponin B2 & $43.2 \pm 1.2$ & $47.8 \pm 1.6$ & $47.7 \pm 0.6$ & $91.6 \pm 3.9$ & $93.9 \pm 3.2$ & $89.5 \pm 3.2$ \\
\hline Tolbutamide (IS) & & $51 \pm 2.3$ & & & $112.7 \pm 2.9$ & \\
\hline
\end{tabular}

compound samples directly dissolved by solvents at the same concentration.

Data analysis

A non-compartmental analysis utilizing WinNonlin software (Pharsight 6.2, NC, USA) with sparse algorithm was applied for the PK parameters calculation. An unpaired Student's $t$-test was used to assess the significance of the in vitro results.

Liver extraction ratio (ER) indicated the fraction of hepatic clearance and first-pass effect, and it was calculated as follows:

$E R=\left(A U C_{\text {por }}-A C_{\text {sys }}\right) / A U C_{\text {por }}$.

Where $A \cup C_{\text {por }}$ and $A U C_{\text {sys }}$ correspond to the areas under the concentration-time curves of compounds in the portal vein plasma and systemic plasma, respectively.

AUC_D was used for evaluating the utilized efficacy per unit in the herb, and it was calculated as follows:

AUC_D = AUC/Dose.
Where AUC represents the area under the concentration-time curves of compounds in vivo, and dose represents the relevant oral dosage of compounds in rats on the herb treatments.

\section{RESULTS}

Method validation

The full validation, including of selectivity, linearity, accuracy, precision, matrix effect, extraction recovery, and stability, was performed for the simultaneous determination of three xanthones and three saponins in the plasma and liver matrix by HPLC-MS/ MS.

The xanthones of neomangiferin, mangiferin, and norathyriol were detected at 5.58,5.91, and $6.80 \mathrm{~min}$, respectively, and the saponins of timosaponin B2, timosaponin B3 and timosaponin A3 were observed at $6.16,6.87$, and $8.68 \mathrm{~min}$, respectively, along with tolbutamide (IS) at $8.27 \mathrm{~min}$ (Fig. 2). Neither endogenous nor exogenous interferences were found at the retention time of those analytes in the biological samples.

The calibration curve of analytes exhibited an excellent linearity with a correlation coefficient $\left(r^{2}\right)$ higher than 0.99, over the 
Table 4. Results of stability analysis for six ingredients in Zhimu in rat plasma $(n=5)$

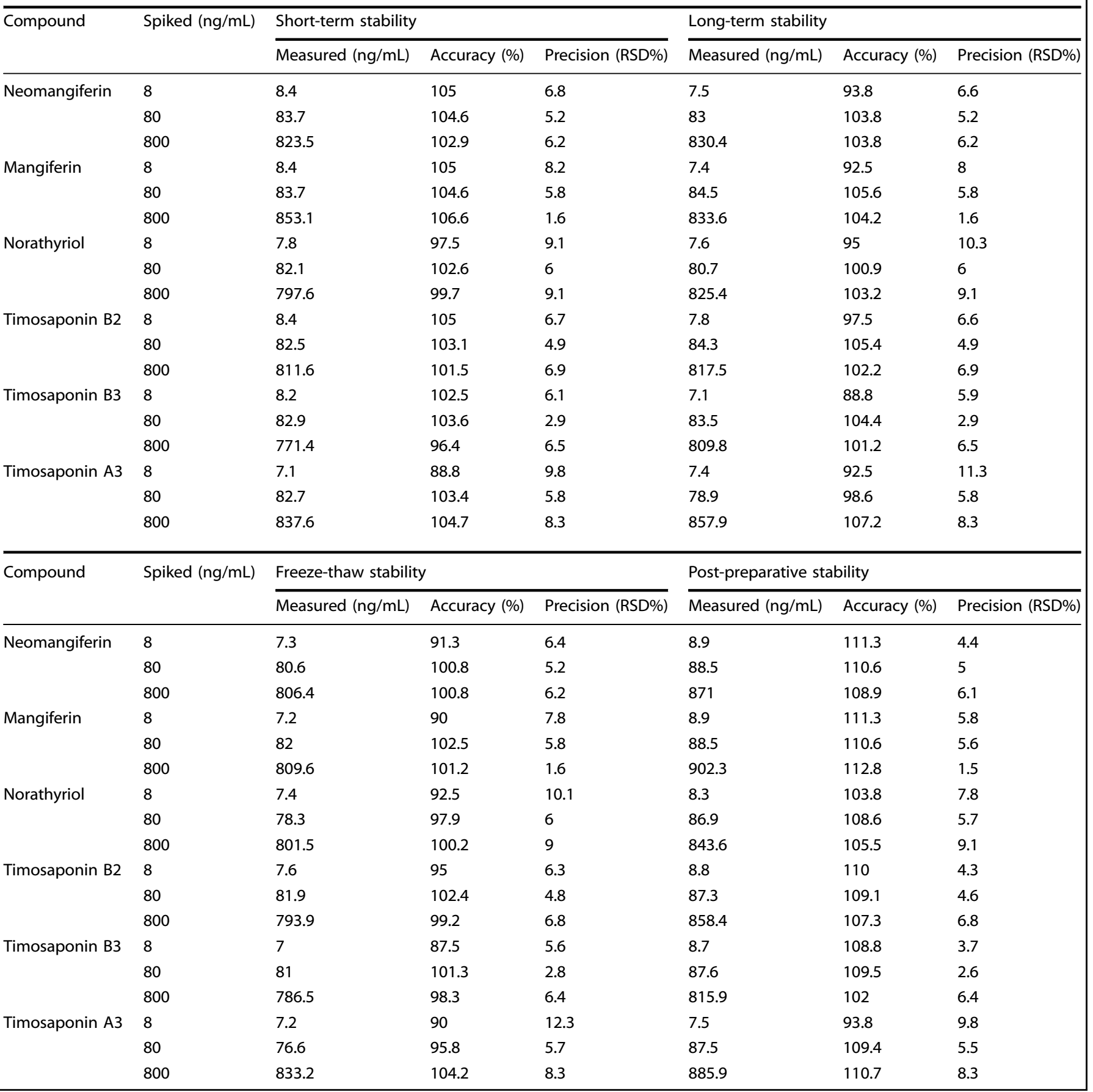

concentration ranged from $3-1000 \mathrm{ng} / \mathrm{mL}$ in the plasma samples. The regression equations were as follows: $Y=0.042415 \times-0.000492$ $\left(r^{2}=0.9931\right) \quad$ for neomangiferin, $Y=0.077848 \times-0.020584$ $\left(r^{2}=0.9902\right)$ for mangiferin, $Y=0.021752 \times-0.003810\left(r^{2}=0.9921\right)$ for norathyriol, $Y=0.006466 \times+0.001473\left(r^{2}=0.9916\right)$ for timosaponin B2, $Y=0.042993 \times-0.004487\left(r^{2}=0.9913\right)$ for timosaponin B3, and $Y=0.004918 \times+0.002913\left(r^{2}=0.9929\right)$ for timosaponin A3.

Both of the intra-day and inter-day precision (RSD) of six analytes at three levels were no more than $11.4 \%$, and the corresponding accuracy ranged from $88 \%$ to $112.7 \%$ in the plasma samples (Table 2).

The mean extraction recovery of each analyst was similar at low, middle and high levels in the plasma samples, and those were
$69.4 \%-71.2 \%$ for neomangiferin, $53.1 \%-61.2 \%$ for mangiferin, $45 \%-54.6 \%$ for norathyriol, $41.4 \%-46 \%$ for timosaponin B2, $56.5 \%-59.4 \%$ for timosaponin B3, and $35.1 \%-41.7 \%$ for timosaponin $\mathrm{A} 3$, along with $51 \% \pm 2.3 \%$ for IS respectively (Table 3 ). All of the matrix effect of analysts were at the range of $85.1 \%-110.1 \%$ at low, middle and high levels, and this was $112.7 \% \pm 2.9 \%$ for IS, suggesting neither ion suppression nor enhancement occurred in the plasma samples.

The analysts in the plasma samples remained stable after storage for $4 \mathrm{~h}$ at ambient temperature, $-80^{\circ} \mathrm{C}$ for 2 weeks, $12 \mathrm{~h}$ post-preparative and three freeze-thaw cycles, evidenced by the accuracy of six analysts within the range of $87.5 \%-112.8 \%$, and the precision below $12.3 \%$ at each level (Table 4 ). 
a

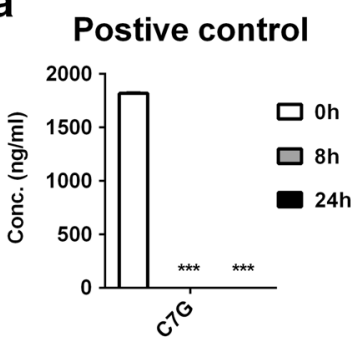

b

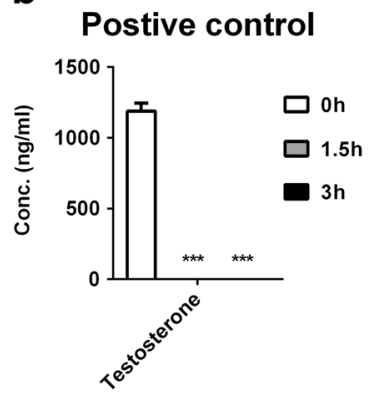

Xanthones

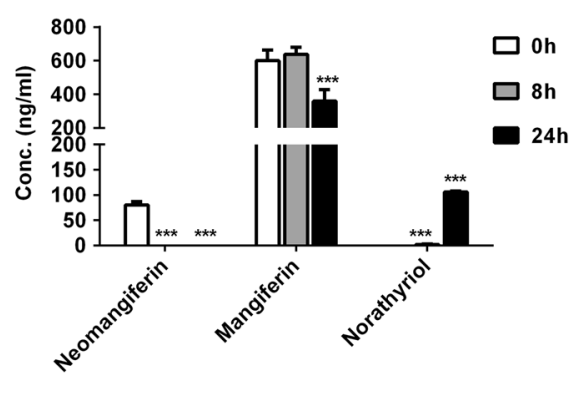

Xanthones

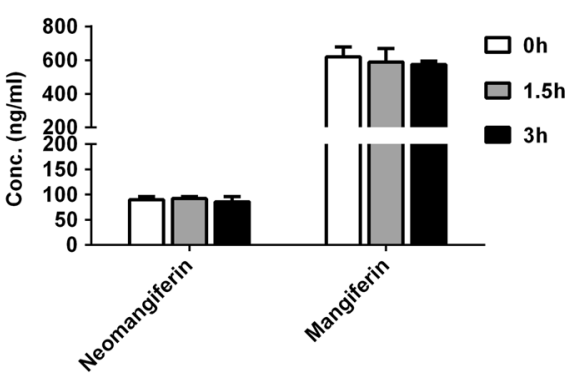

Saponins

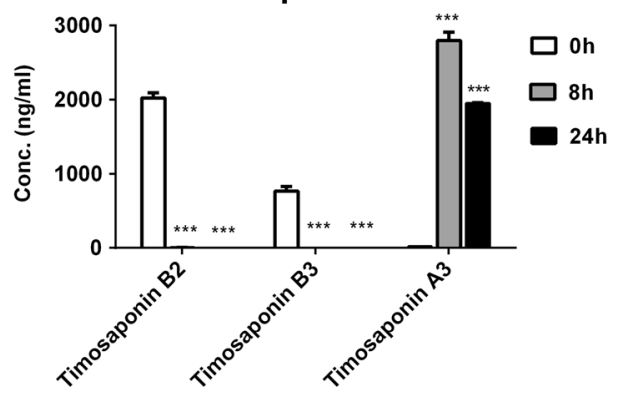

Saponins

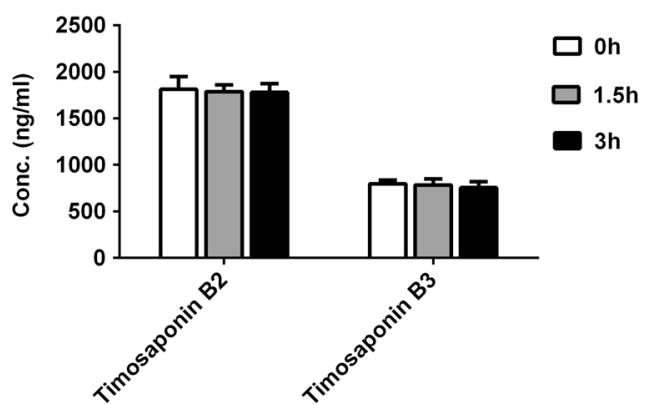

Fig. 3 Evaluation the microbiota-mediated metabolism of xanthones and saponins in Zhimu decoction in the enterobacteria samples after incubation of Zhimu decoction at 0, 8, and $24 \mathrm{~h}, \mathrm{C7G}$ used as a positive control (a). Evaluation of liver-mediated metabolism of xanthones and saponins in the rat primary hepatocytes after incubation of Zhimu decoction $0,1.5$, and $3 \mathrm{~h}$, testosterone used as a positive control (b). ${ }^{*} P<0.05,{ }^{* *} P<0.01,{ }^{* * *} P<0.001$ compared with the control

As with the plasma matrix, full validations were investigated and verified in the liver samples. Consequently, these results met the demands for the accurate determination of six ingredients in the liver matrix (seen in the supplementary materials). Those results confirmed the favorable availability for the simultaneous determination of three xanthones and three saponins in the plasma and liver matrix by HPLC-MS/MS.

Determination of xanthones and saponins in the Zhimu decoction Among the ingredients, timosaponin B2 was of the highest content at the concentration of $18,150 \mu \mathrm{g} / \mathrm{mL}$ in the Zhimu decoction $(0.7 \mathrm{~g} / \mathrm{mL})$, followed by mangiferin, timosaponin $\mathrm{B} 3$, and neomangiferin at 6100,4790 , and $840 \mu \mathrm{g} / \mathrm{mL}$, respectively. However, the content of timosaponin $\mathrm{A} 3$ remained extremely low at $97 \mu \mathrm{g} / \mathrm{mL}$, only accounting for $0.5 \%$ of that of timosaponin B2, along with an undetected amount of norathyriol in the Zhimu decoction. When rats taking the Zhimu decoction at the oral dosage of $7 \mathrm{~g} / \mathrm{kg}$, it was equivalent to timosaponin B2, mangiferin, timosaponin B3, and neomangiferin at the oral dosage of 181.5, $61.0,47.9$, and $8.4 \mathrm{mg} / \mathrm{kg}$, respectively, when the oral dosage of timosaponin A3 and norathyriol can be negligible with $<1 \mathrm{mg} / \mathrm{kg}$ on Zhimu treatments.

In vitro study

After incubation for $8 \mathrm{~h}$, the positive control (C7G, $20 \mu \mathrm{g} / \mathrm{mL})$ was completely metabolized in the enterobacteria samples, indicating of the excellent applicability for evaluating microbiota-mediated metabolism (Fig. 3a). Regarding xanthones, after incubation of Zhimu decoction, neomangiferin were all metabolized, and the levels of mangiferin elicited a bit increased trend $(P>0.05)$, and those decreased with difference $(P<0.001)$ until incubation for $24 \mathrm{~h}$ (Fig. 3a). This was due to neomangiferin was metabolized into mangiferin firstly by lose of one glucose unit, and then mangiferin was converted into norathyriol after elimination of the other glucose group. Correspondingly, norathyriol, the aglycone of neomangiferin and mangiferin, was generated after incubation of
Zhimu decoction for $8 \mathrm{~h}$, and an apparently increased concentration happened by hundreds of times during the incubated time range of $8-24 \mathrm{~h}$ (Fig. 3a). As for saponins, similar to xanthones, after incubation of Zhimu decoction for $8 \mathrm{~h}$, timosaponin B2 and timosaponin B3 were all metabolized, corresponding to the dramatically increased concentration of timosaponin A3 by 218fold during the incubated time for $8 \mathrm{~h}$, although with slight decrease by $8.8 \%$ during the time range of $8-24 \mathrm{~h}$ (Fig. 3a).

The positive control $(10 \mu \mathrm{mol} / \mathrm{L}$ testosterone) was all metabolized in the primary hepatocytes within $1.5 \mathrm{~h}$, thus confirming the excellent metabolic activity of those cells (Fig. 3b). However, there is no obvious change on the concentration of all the prototypes, involved of neomangiferin, mangiferin, timosaponin B2, and timosaponin B3, before and after Zhimu decoction incubation ( $P$ $>0.05$ ), indicating of the limited metabolic activity for those ingredients in Zhimu in the liver (Fig. 3b).

Briefly, xanthones, including of neomangiferin, mangiferin, and saponins, involved of timosaponin B2 and timosaponin B3, could be respectfully transformed into norathyriol and timosaponin $A 3$ via the microbiota-mediated metabolism, but those were limited to the liver-mediated metabolism.

\section{In vivo study}

The established and validated HPLC-MS/MS method was successfully applied to the determination of xanthones and saponins in the portal vein plasma, liver, and systemic plasma, following oral administration of Zhimu decoction at the dosage of $7 \mathrm{~g} / \mathrm{kg}$ (Figs. 4, 5; Table 5).

PK before and after hepatic disposition

Following oral administration of Zhimu decoction, three xanthones and three saponins were all accurately determined in the portal vein plasma and systemic plasma, and the compounds were of higher levels in the former (Fig. 4a). Among the xanthones, mangiferin was of the greatest exposure both in the portal vein plasma and systemic plasma, where the AUCs of mangiferin were 
a

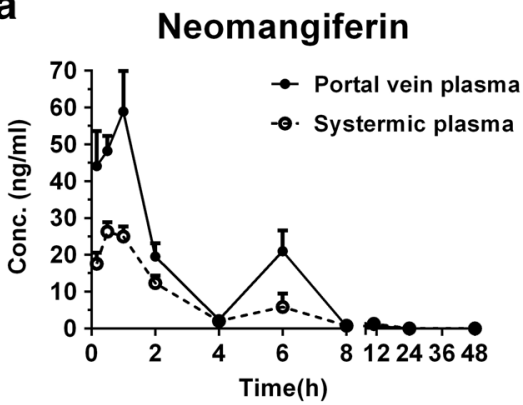

Timosaponin B2

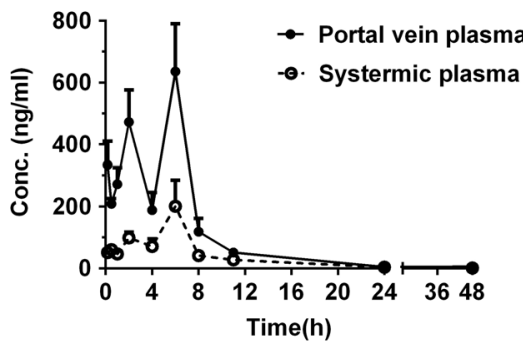

b

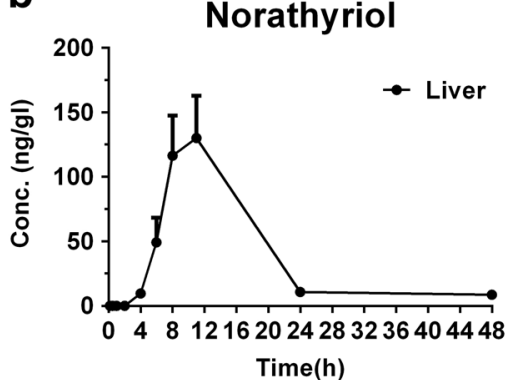

Mangiferin

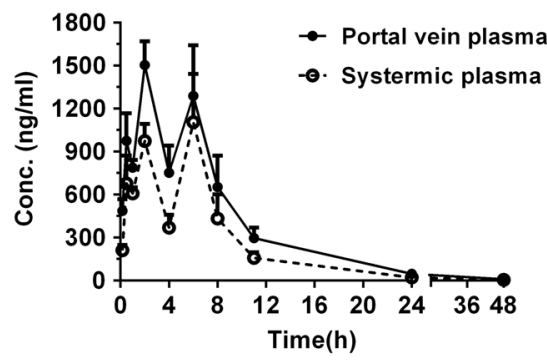

Timosaponin B3

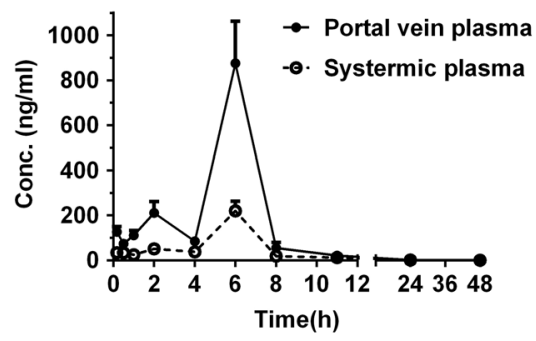

Timosaponin A3

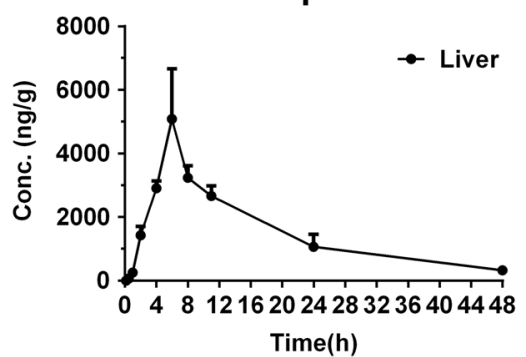

Norathyriol

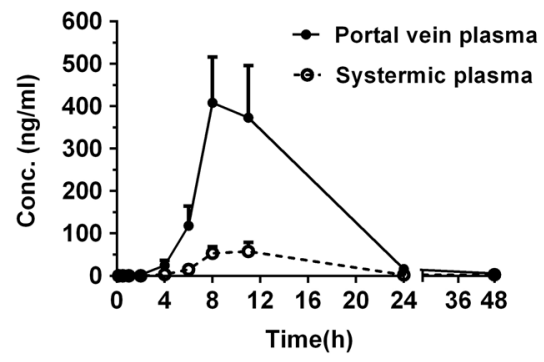

Timosaponin A3

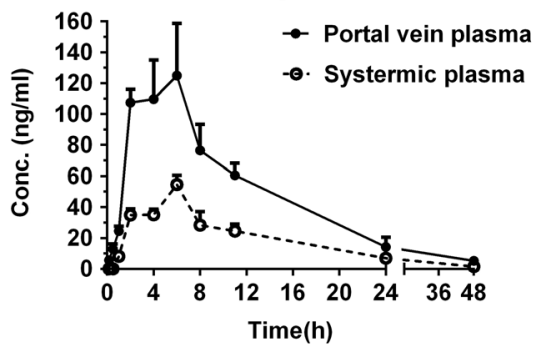

Fig. 4 Mean concentration-time curves of the xanthones and saponins in the portal vein plasma and systemic plasma (a) and liver (b), after oral administration of Zhimu decoction at $7 \mathrm{~g} / \mathrm{kg}$ in rats $(n=5)$

at $\quad 12,434.4 \pm 1106.1 \mathrm{ng} / \mathrm{mL} / \mathrm{h}$, and $8008.9 \pm 1148.7 \mathrm{ng} / \mathrm{mL} / \mathrm{h}$, respectively, corresponding to its ER at $35.6 \%$. Although no detection in the Zhimu decoction, there were substantial exposure of norathyriol in the portal vein plasma with the AUC at $4658.6 \pm$ $874 \mathrm{ng} / \mathrm{mL} / \mathrm{h}$, and after undergoing serious hepatic clearance with the ER at $84.9 \%$, the AUC of norathyriol was at $705.2 \pm 133.1 \mathrm{ng} /$ $\mathrm{mL} / \mathrm{h}$ in the systemic plasma. Neomangiferin was of the lowest levels with the AUC only at $154 \pm 13.9$ and $72.3 \pm 9.5 \mathrm{ng} / \mathrm{mL} / \mathrm{h}$ in the portal vein plasma and systemic plasma, along with its ER at $53 \%$. Likewise, the order of $C_{\max }$ from high to low was mangiferin, norathyriol, and neomangiferin both in the portal vein plasma and systemic plasma. There was an observable multiple-peak phenomenon for mangiferin and neomangiferin, but not for norathyriol in the time-concentration curves. The first $T_{\max } \mathrm{s}$ of neomangiferin and mangiferin happened at the time range of $0.5-2 \mathrm{~h}$, and the second ones occurred at $6 \mathrm{~h}$ both in the portal vein plasma and systemic plasma. The $T_{\max } s$ of norathyriol happened at 8 and $11 \mathrm{~h}$ in the portal vein plasma and systemic plasma, respectively, probably associated with the slow metabolized rate of xanthones on the effect of enterobacteria (Fig. 3a). Except of neomangiferin with $T_{1 / 2}$ at about $2 \mathrm{~h}$, those for mangiferin and norathyriol were at the time range of $6.4-10 \mathrm{~h}$ both in the portal vein plasma and systemic 331 plasma.

Similarly, the levels of saponine were obviously higher in the portal vein plasma than those in the systemic plasma, including timosaponin B2 (3580.6 \pm 373.5 vs. $1163.7 \pm 197.4 \mathrm{ng} / \mathrm{mL} / \mathrm{h})$, timosaponin B3 $(2730.2 \pm 357.3$ vs. $808.7 \pm 109.1 \mathrm{ng} / \mathrm{mL} / \mathrm{h})$, timosaponin A3 $(1656.7 \pm 121.1$ vs. $650.5 \pm 45.2 \mathrm{ng} / \mathrm{mL} / \mathrm{h})$. Those corresponded to the severe liver first-pass effect of those saponins, whose ERs were at the range of $60.7 \%-70.4 \%$. Timosaponin B2 was of the highest AUC in both the portal vein plasma and systemic plasma. The $C_{\max }$ of timosaponin $B 3$ was a bit higher than that of timosaponin B2 $(875.1 \pm 187 \mathrm{ng} / \mathrm{mL}$ vs. $635.7 \pm$ $155.5 \mathrm{ng} / \mathrm{mL}$ ) in the portal vein plasma, and those were very similar in the systemic plasma $(200.7 \pm 85.1$ vs. $220.1 \pm 43.7 \mathrm{ng} /$ $\mathrm{mL}$ ). Timosaponin A3 was of the lowest $C_{\max }$ and AUC in both the portal vein plasma and systemic plasma. There was a significant multi-peak phenomenon in the time-concentration curves of timosaponin B2 and timosaponin B3, while that remained obscure to timosaponin $A 3$ in both portal vein plasma and systemic plasma. The $T_{\max } \mathrm{s}$ of those saponins all appeared at $6 \mathrm{~h}$. The $T_{1 / 2}$ of timosaponin B3 was at $7.9 \mathrm{~h}$, a bit smaller than those of timosaponin B2 and timosaponin A3 at 9.5 and $10.3 \mathrm{~h}$, respectively.

After normalizing the AUC by the oral dosage, the AUC_D of mangiferin was the maximum, followed by timosaponin B3, timosaponin B2, and neomangiferin in both the portal vein plasma and systemic plasma. This reflected that among the ingredients in Zhimu, mangiferin exerted the highest utilized efficacy in the plasma both before and after hepatic clearance following oral administration.

Liver distribution

Only the metabolites- norathyriol and timosaponin A3, but none of any prototypes, were observed in the liver. The levels of 

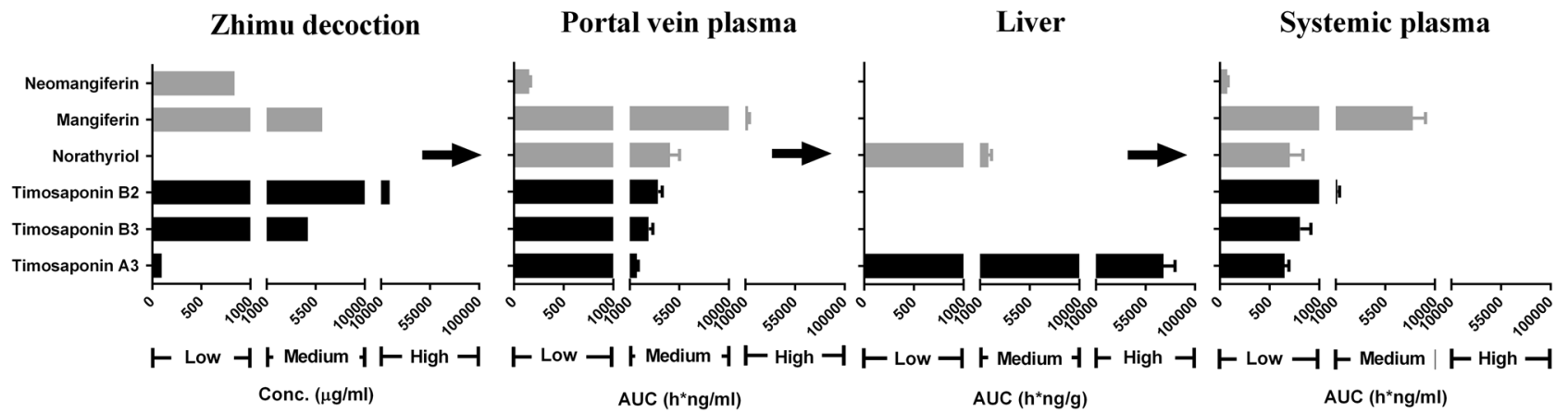

Liver

Systemic plasma

Fig. 5 Comparison on the $A U C_{S}$ of the xanthones and saponins in the portal vein plasma, liver and systemic plasma after oral administration of Zhimu decoction at $7 \mathrm{~g} / \mathrm{kg}$, along with the content in the herb

timosaponin A3 were as high as $71,386 \pm 10,285.1 \mathrm{ng} / \mathrm{g} / \mathrm{h}$, about 40.7 -fold of that of norathyriol at $1754.4 \pm 288.8 \mathrm{ng} / \mathrm{g} / \mathrm{h}$ (Fig. $4 \mathrm{~b}$ ). Similarly, the $C_{\max }$ of timosaponin $A 3$ was 39.1-time of that of norathyriol. The $T_{\max } \mathrm{s}$ of norathyriol and timosaponin A3 occurred at 11 and $6 \mathrm{~h}$, respectively, probably correlated with the slow metabolized rate for xanthones and saponins on the effect of enterobacteria (Fig. 3a). The $T_{1 / 2} \mathrm{~S}$ of timosaponin $\mathrm{A} 3$ and norathyriol were a little long at 12.1 and $10.6 \mathrm{~h}$, respectively.

Given their negligible content in the herb, as well as the microbiota-mediated metabolism of xanthone and saponin prototypes can generate norathyriol and timosaponin A3 (Fig. 3a), the substantial hepatic exposure of timosaponin $\mathrm{A} 3$ and norathyriol was mainly attributed to the microbiota-mediated metabolism of prototypes, rather than direct absorption from the herb. This was also consistent with the non-ignorable levels of norathyriol and timosaponin A3 in the portal vein plasma, by which they were transported to the liver after microbiotamediated metabolism and gut absorption.

\section{DISCUSSION}

Owing to the inherent nature containing multiple ingredients, the herbal medicine exerts the therapeutic efficacy or toxicity as a whole, probably involved of synergistic, additive or antagonistic effect via the complicated DDls [31]. Hence, it is well acknowledged that there may be grand disparity on the efficacy or safety between the herb and pure compound treatments. Serving as the material base for comprehensively understanding the herbal efficacy or safety, the primary work is to make it clear of the in vivo dynamic disposition of main prototypes and metabolites in a holistic way, particularly with the observation of the toxic substances. Hence, via the in vitro and in vivo combination, the metabolism, pharmacokinetics and hepatic disposition of xanthones and saponins was comprehensively clarified on Zhimu treatments in this study.

Given the giant variation of the chemical structures among the analytes in Zhimu (Fig. 1), much efforts were made for the optimization for the simultaneous determination of three xanthones and three saponins in Zhimu, including of verification of matrix inference between the positive and negative mode, comparison of sensitivity between organic solvent precipitation and liquid-liquid extraction, determination of precipitator (acetonitrile/methanol), the choice on the acidity of acetonitrile (1\%-10\% of formic/acetic acid) and confirmation of appropriate internal standard (IS). Finally, acetonitrile containing 3\% acetic acid precipitation, negative MRM mode, and tolbutamide as internal standard were employed, considering the comprehensive benefits of high sensibility, less matrix inference and perfect peak shapes.

Timosaponin A3 has arisen attention because of the high potentials on anticancer, anti-dementia and antiplatelet aggradation [32-34]. However, we previously discovered timosaponin A3
$(100 \mathrm{mg} / \mathrm{kg}$ per day) significantly increased the levels of alanine aminotransferase (ALT) and total bile acid (TBA), the typical pathological indicators of cholestasis, after continuous oral administration of timosaponin A3 for 14 days [22]. Rather than directly inhibiting the bile acid transporters, timosaponin A3 disrupted the polarization of the bile acid transporter system by generating reactive oxygen species (ROS) [22]. Excessive ROS can oxidize the proteins, DNA, lipid and other macromolecules, resulting in destroying the cellular physiological structure and causing hepatotoxicity [35]. In the presented study, the content of timosaponin A3 was too low in the Zhimu decoction to be taken into consideration, which only accounted for less than $0.5 \%$ of that of timosaponin B2 in the herb decoction. However, given the in vitro results that timosaponin B2 and timosaponin B3 were metabolized entirely into timosaponin $\mathrm{A} 3$ through deglycosylation in the enterobacteria samples (Fig. 3a), timosaponin A3 may exist as metabolites in vivo. In fact, following oral administration of Zhimu decoction at the dosage of $7 \mathrm{~g} / \mathrm{kg}$, there was substantial exposure of timosaponin A3 in the portal vein plasma, corresponding to the in vitro results that the generation of timosaponin A3 contributed to the microbiota-, but not liver-, mediated metabolism of other saponins in Zhimu (Fig. 3). After transported by the portal vein plasma, timosaponin $A 3$ elicited the $C_{\max }$ as high as $5085.3 \pm 1581.5 \mathrm{ng} / \mathrm{g}$ (about $7 \mu \mathrm{M}$ ) in the liver (Fig. 4). It should be alert that timosaponin $\mathrm{A} 3$ even at $5 \mu \mathrm{M}$ can obviously induce oxidative stress and downregulate bile acid transporters in the hepatocytes [22]. In addition to the microbiota-regulated conversion from other saponins in Zhimu, the substantial hepatic exposure of timosaponin $A 3$ was also attributed to its high membrane permeability resulting from its high hydrophobicity ( $\log P$ at $2.978 \pm 0.884)$, and low bile excretion with the bile excretion index (BEI) only at $12.8 \%$ (data not shown). After the hepatic clearance, just $39.3 \%$ of timosaponin A3 from the portal vein plasma got into the circulating system. The serious liver firstpass effect of timosaponin A3 (ER at $60.7 \%$ ) corresponded to the sizable levels in the liver, but not with the limited hepatic metabolism of timosaponin A3, according to the incubated results in the primary hepatocytes (Figure S2). Notably, timosaponin A3 also elicited slow absorption and elimination rate in the liver, where the $T_{1 / 2}$ and $T_{\max }$ of timosaponin A3 were at 12.1 and $6 \mathrm{~h}$, respectively (Table 5 ). This indicated that there was very likely to be the high hepatic accumulation of timosaponin A3 after continuous oral administration of Zhimu.

Interestingly, in contrast to the substantial exposure of timosaponin $A 3$, there were no detectable levels of timosaponin B2 and timosaponin B3, the saponins with high amounts in Zhimu, in the liver (Fig. 5). On the one hand, this was associated with the microbiota-mediated metabolism of timosaponin B2 and timosaponin B3 (Fig. 3a). One the other hand, given the chemical structures containing one more glucose molecule (Fig. 1), timosaponin B2 and timosaponin B3 exerted poorer membrane 
Table 5. The PK parameters of the xanthones and saponins in the portal vein plasma, liver and systemic plasma after oral administration of Zhimu decoction at $7 \mathrm{~g} / \mathrm{kg}$ in rats $(n=5)$

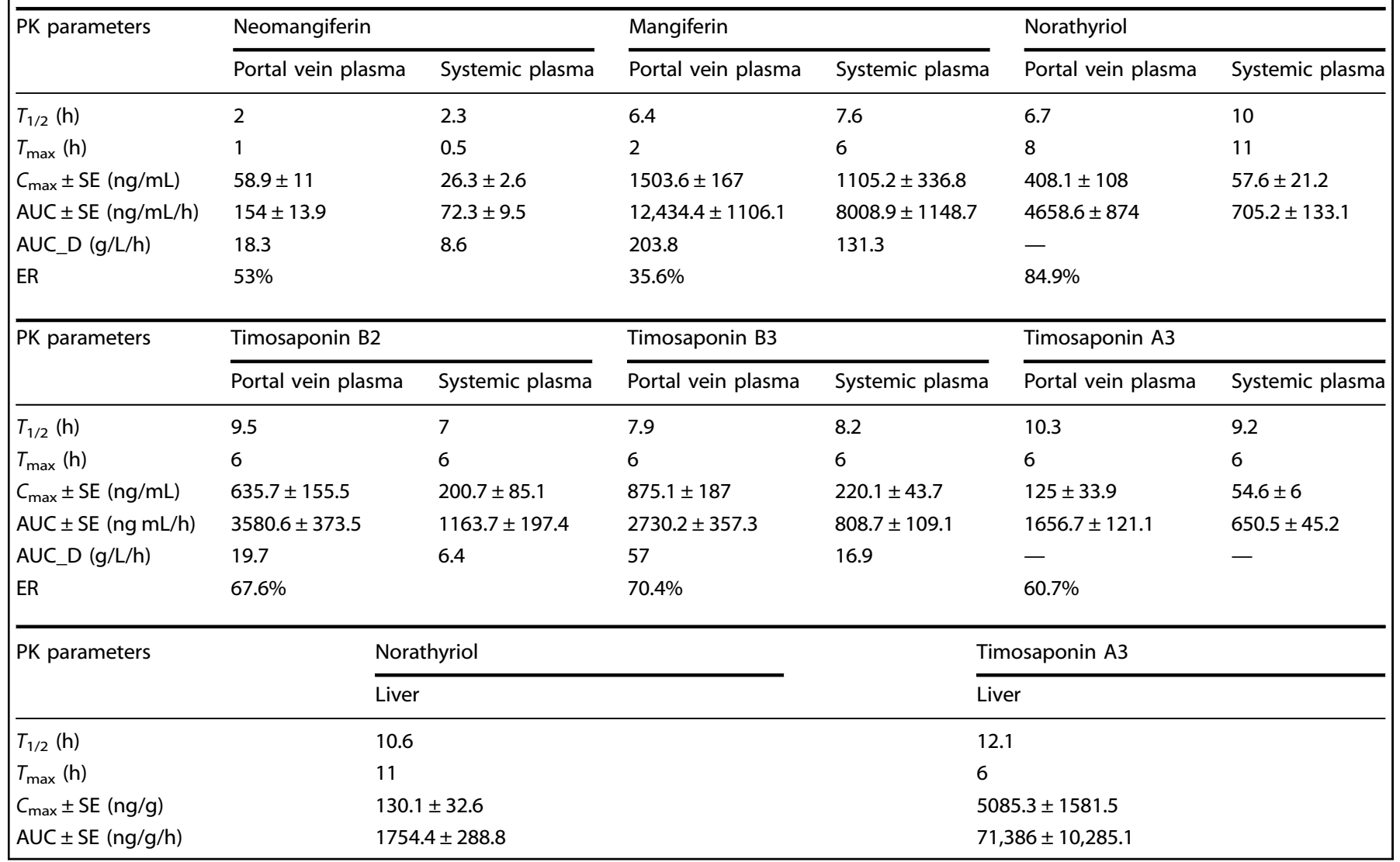

permeability than timosaponin A3, resulting in the limited passive diffusion in the liver. In spite of the limited hepatic accumulation (Fig. 5) and metabolism (Fig. 3b), timosaponin B2 and timosaponin B3 underwent severe liver first-pass effect, whose ER was at $67.6 \%$ and $70.4 \%$, respectively. Those were probably associated with their bile excretion, based on our previous conclusion that timosaponin B2 was the substrate of breast cancer resistance protein (Bcrp) and multidrug resistance-associated protein 2 (Mrp2), the efflux transporters responsible for the bile excretion, in line with the BEI of timosaponin B2 at 44\% [21], although those for other ingredients awaited further study. Given the limited hepatic exposure and no reports regarding the anti-oxidation, timosaponin B2, and timosaponin B3 were likely to have insignificant role in the whole hepatoprotective activity on Zhimu treatments.

Mangiferin, a well-established naturally antioxidant, can neutralize various reactive species and regulate the expression and activity of key detoxification enzymes, involved of superoxide, catalase, and glutathione-S-transferase [36]. Compared to the frequently used antioxidants, such as vitamin $C$, vitamin $E$ and $\beta$ carotene, mangiferin showed preferable or comparative antioxidative capacity in vivo [25]. In our previous study, mangiferin at $25 \mu \mathrm{M}$ can completely restore the ROS, ATP and LDH induced by timosaponin $A 3$ to the normal levels in hepatocytes, and such an astonishing effect even can not be achieved on $\mathrm{N}$-acetyl-L-cysteine treatments [22]. To our surprise, although with the highest exposure in the portal vein plasma, there were limited exposure of mangiferin in the liver (Fig. 5). This was in accord with our previous study that there were limited exposure of mangiferin in the liver, which was highly associated to the poor membrane permeability of mangiferin, following oral intake of mangiferin even at the high dosage of $200 \mathrm{mg} / \mathrm{kg}$ in animals [19]. However, some researchers have documented that mangiferin can efficiently resist the oxidative stress-mediated liver dysfunction and exerted the hepatoprotective activity with the oral dosage of mangiferin no more than $100 \mathrm{mg} / \mathrm{kg}$ in animals $[25,37,38]$. Thus, based on the discrepancy of significant efficacy and limited exposure in liver, it was reasonably deduced that there might be some specific sites, but not liver, where mangiferin took action as antioxidants and indirectly caused the liver protection on mangiferin treatments. Analogously, in views of the maximum exposure in the circulating system (Fig. 5), mangiferin was very likely to be carried to the specific organs where it took actions of anti-oxidation and fighting against timosaponin A3-induced hepatotoxicity indirectly on Zhimu treatments. Besides, according to our previous study, compared to the pure compound treatments, the AUC of mangiferin was increased by 11.5 -fold in the plasma on the Zhimu decoction treatments, because of drug-drug interaction (DDI) of timosaponin B2 and mangiferin at pharmacokinetic levels [20]. This also potentially explained why mangiferin elicited the maximum AUC and AUC_D in the plasma on herb treatments, although the bioavailability of mangiferin was only at $1.2 \%$ [39]. Given the $\mathrm{C} 7-\mathrm{OH}$ position occupied by a glucose molecule, neomangiferin should have relatively poor oxidation resistance and make an ignorable contribution to the whole antioxidant ability of Zhimu with its low exposure in vivo (Fig. 5).

Interestingly, in contrast to no observation of xanthone prototypes, norathyriol, the aglycone of mangiferin, showed certain hepatic accumulation (Fig. 5) on Zhimu treatments. This was in contrast to no observation of norathyriol on mangiferin treatments alone [19], probably due to the timosaponin B2 and mangiferin interaction leading to the increased exposure of 
norathyriol [20], as well as the generation of norathyriol from neomangiferin except of mangiferin (Fig. 3a), on Zhimu treatments. Similar to that of timosaponin A3, the exposure of norathyriol in vivo was attributed to the microbiota-, but not liver-, mediated metabolism of xanthones in Zhimu (Fig. 3). In agreement with generation of norathyriol via microbiota-mediate metabolism, there was also non-ignorable levels of norathyriol in the portal vein plasma, where the AUC of norathyriol took up of $37.4 \%$ of those of mangiferin. Nevertheless, the levels of norathyriol only accounted for $8.8 \%$ of those of mangiferin in the systemic plasma. Correspondingly, the ER of norathyriol was as high as $84.9 \%$, and this was correlated with the substantial hepatic accumulation (Fig. 5) and obvious hepatic metabolism of norathyriol (Figure.S2). Moreover, via the comparison on their $T_{\max } \mathrm{S}$ and $T_{1 / 2} \mathrm{~S}$, norathyriol elicited similar absorption and elimination rate to timosaponin A3 in the liver. Those implied the comparable probability of hepatic accumulation of timosaponin A3 also happened to norathyriol on repeated Zhimu treatments. As reported, norathyriol was of similar antioxidative capacity to mangiferin with the $\mathrm{IC}_{50}$ both at $9 \mu \mathrm{M}$ [37]. This was also in line with the conclusion that $\mathrm{B}$-ring, $6-\mathrm{OH}$ and $7-\mathrm{OH}$ on the chemical structure of xanthones made the most significant contribution to the antioxidative ability, according to the theoretical bond dissociation enthalpy (BDE) analysis [40]. Thus, norathyriol, as a powerful antioxidant in the liver, was likely to have a direct role in fighting against timosaponin A3-induced hepatotoxicity. Given the levels of norathyriol only accounted up of $2.5 \%$ of those of timosaponin A3 in the liver, more studies are still in demand on the quantitative relationship of timosaponin A3 and norathyriol on balancing hepatic status between oxidation and anti-oxidation in the future. Besides, compared to the mangiferin treatments, the DDI between mangiferin and timosaponin B2 also cause the increased exposure of norathyriol by 5.9folds in the plasma on the Zhimu treatments [20]. Thus, such a DDI should also contribute indirectly to the whole anti-oxidation of Zhimu via improving the levels of mangiferin and norathyriol in vivo.

In spite of limited content in the herb, timosaponin A3, who can induce the hepatotoxicity via oxidative stress, elicited substantial hepatic exposure as the microbiota-mediated metabolite of other saponins in Zhimu, following oral administration of Zhimu in rats. To sum up, the discrepancy of the safe clinical medication of Zhimu and timosaponin A3-induced hepatotoxicity can be exploratively interpreted by the presence of antioxidants in vivo. Norathyriol, the microbiota-mediated metabolites of xanthones with specific hepatic exposure, should have a direct role in fighting against the oxidative stress induced by timosaponin $A 3$ in the liver. Mangiferin, a xanthone with the maximum exposure in the plasma, may take the action of as antioxidants in some specific sites and exerted the hepatoprotective activity indirectly. The DDI of timosaponin B2 and mangiferin at pharmacokinetic levels could also make significant contribution indirectly to the overall antioxidation of Zhimu by increasing the in vivo levels of mangiferin and norathyriol. More scientific evaluation is still urgently required to explore the quantitative relations in vivo between antioxidation generated by xanthones and oxidative stress induced by timosaponin A3. Notably, given the sizable levels and slow elimination rate of timosaponin $\mathrm{A} 3$ in the liver, more attention should be paid to the safety on the continuous clinical medication of Zhimu in the future.

\section{ACKNOWLEDGEMENTS}

The current study was supported by The National Natural Science Foundation of China (Nos. 81030065 and 81374052), National Natural Science Foundation for Young Scientists of China (Nos. 81603280 and 81602998), and Strategic Priority Program of Chinese Academy of Sciences (No. XDA12040203).

\section{AUTHOR CONTRIBUTIONS}

Y.X., Z.X., X.-t.T. and C.-g.H. were responsible for the research design; Y.X., Z.X., P.H. and M.-C.C. conducted the experiments; Z.-I.S. and Y.-r.X. contributed new reagents or analytical tools; Y.X., X.-t.T. and C.-g.H. performed data analysis and wrote the manuscript.

\section{ADDITIONAL INFORMATION}

Supplementary information accompanies this paper at https://doi.org/10.1038/ 441401-018-0012-z.

Competing interests: The authors declare no competing interests.

Publisher's note: Springer Nature remains neutral with regard to jurisdictional claims in published maps and institutional affiliations.

\section{REFERENCES}

1. Bunchorntavakul C, Reddy KR. Review article: herbal and dietary supplement hepatotoxicity. Aliment Pharmacol Ther. 2013;37:3-17.

2. Stickel F. Liver injury from herbal and dietary supplements. Dtsch Med Wochenschr. 2015;140:908-11.

3. Ng AWT, Poon SL, Huang MN, Lim JQ, Boot A, Yu W, et al. Aristolochic acids and their derivatives are widely implicated in liver cancers in Taiwan and throughout. Asia Sci Transl Med. 2017;9:eaan6446.

4. Che CT, Wang ZJ, Chow MS, Lam CW. Herb-herb combination for therapeutic enhancement and advancement: theory, practice and future perspectives. Molecules. 2013;18:5125-41.

5. lida $Y$, Oh KB, Saito $M$, Matsuoka $H$, Kurata $H$, Natsume $M$, et al. Detection of antifungal activity in Anemarrhena asphodeloides by sensitive BCT method and isolation of its active compound. J Agric Food Chem. 1999:47:584-7.

6. Zhang J, Zhang M, Sugahara K, Sagara Y, Meng Z, Xu S, et al. Effect of steroidal saponins of Anemarrhenae rhizoma on superoxide generation in human neutrophils. Biochem Biophys Res Commun. 1999;259:636-9.

7. Han J, Yang N, Zhang F, Zhang C, Liang F, Xie W, et al. Rhizoma anemarrhenae extract ameliorates hyperglycemia and insulin resistance via activation of AMP-activated protein kinase in diabetic rodents. J Ethnopharmacol. 2015;172:368-76.

8. Du H, Zhao H, Lai X, Lin Q, Zhu Z, Chai Y, et al. Metabolic profiles revealed synergistically anti-depressant effects of lilies and Rhizoma anemarrhenae in a rat model of depression. Biomed Chromatogr. 2016;31:e3923.

9. Wang Y, Dan Y, Yang D, Hu Y, Zhang L, Zhang C, et al. The genus Anemarrhena bunge: a review on ethnopharmacology, phytochemistry and pharmacology. $J$ Ethnopharmacol. 2014;153:42-60.

10. Min Q, Cai X, Sun W, Fei G, Li Z, Zhang Q, et al. Identification of mangiferin as a potential glucokinase activator by structure-based virtual ligand screening. Sci Rep. 2017;7:44681.

11. Fomenko EV, Chi Y. Mangiferin modulation of metabolism and metabolic syndrome. Biofactors. 2016;42:492-503.

12. Li TJ, Qiu Y, Yang PY, Rui YC, Chen WS. B-II improves memory and learning dysfunction induced by cerebral ischemia in rats. Neurosci Lett. 2007:421:147-51.

13. Liu H, Wang K, Tang Y, Sun Z, Jian L, Li Z, et al. Structure elucidation of in vivo and in vitro metabolites of mangiferin. J Pharm Biomed Anal. 2011;55:1075-82.

14. Liu H, Wu B, Pan G, He L, Li Z, Fan M, et al. Metabolism and pharmacokinetics ofmangiferin in conventional rats, pseudo-germ-free rats, and streptozotocininduced diabetic rats. Drug Metab Dispos. 2012;40:2109-18.

15. Fu Z, Li Z, Xue R, Jiang J, Huang C. Stereoisomerism metabolites found in rats after oral administration of timosaponin B-II using HPLC-Q-TOF-MS and NMR methods. Rsc Adv. 2015;5:60650-7.

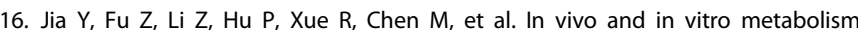
study oftimosaponin B-II using HPLC-ESI-MSn. Chromatographia. 2015;78:1175-84.

17. Li D, Xue R, Li Z, Chen M, Jiang W, Huang C. In vivo metabolism study of timosaponin BIII in rat using HPLC-QTOF-MS/MS. Chromatographia. 2014;77:853-8.

18. Jia $Y$, Wu B, Fan $M$, Wang J, Huang J, Huang $C$. High-performance liquid chromatography-electrospray ionization tandem mass spectrometry for metabolism study of timosaponin All. J Chromatogr Sci. 2014;52:418-22.

19. Tian X, Gao Y, Xu Z, Lian S, Ma Y, Guo X, et al. Pharmacokinetics of mangiferin and its metabolite-norathyriol. Part 1: Systemic evaluation of hepatic first-pass effect in vitro and in vivo. Biofactors. 2016:42:533-44.

20. Tian X, Xu Z, Li Z, Ma Y, Lian S, Guo X, et al. Pharmacokinetics of mangiferin and its metabolite-norathyriol. Part 2: Influence of UGT, CYP450, P-gp, and 
enterobacteria and the potential interaction in Rhizoma anemarrhenae decoction with timosaponin B2 as the major contributor. Biofactors. 2016;42:545-55.

21. Sheng J, Tian X, Xu G, Wu Z, Chen C, Wang L, et al. The hepatobiliary disposition of timosaponin b2 is highly dependent on influx/efflux transporters but not metabolism. Drug Metab Dispos. 2015;43:63-72.

22. Wu ZT, Qi XM, Sheng JJ, Ma LL, Ni X, Ren J, et al. Timosaponin A3 induces hepatotoxicity in rats through inducing oxidative stress and down-regulating bile acid transporters. Acta Pharmacol Sin. 2014;35:1188-98.

23. Panda SS, Chand M, Sakhuja R, Jain SC. Xanthones as potential antioxidants. Curr Med Chem. 2013;20:4481-507.

24. Xia G, Li X, Zhu X, Yin X, Ding H, Qiao Y. Mangiferin protects osteoblast against oxidative damage by modulation of ERK5/Nrf2 signaling. Biochem Biophys Res Commun. 2017;491:807-13.

25. Sanchez GM, Re L, Giuliani A, Nunez-Selles AJ, Davison GP, Leon-Fernandez OS. Protective effects of Mangifera indica L. extract, mangiferin and selected antioxidants against TPA-induced biomolecules oxidation and peritoneal macrophage activation in mice. Pharmacol Res. 2000;42:565-73.

26. Feng Y, Chen BT, Lin AH, Liu YM. Simultaneous determination of timosaponin B-II and A-III in rat plasma by LC-MS/MS and its application to pharmacokinetic study. J Chromatogr B Anal Technol Biomed Life Sci. 2014;965:119-26.

27. Liu Z, Dong X, Ding X, Chen X, Lv L, Li Y, et al. Comparative pharmacokinetics of timosaponin B-II and timosaponin A-III after oral administration of Zhimu-Baihe herb-pair, Zhimu extract, free timosaponin B-II and free timosaponin A-III to rats. J Chromatogr B Anal Technol Biomed Life Sci. 2013;926:28-35.

28. Xie $Y$, Wang $X$, Wang $S$, Wang $Y$, Tian $H$, Yuan $Y$, et al. Metabolism and pharmacokinetics ofmajor polyphenol components in rat plasma after oral administration of total flavonoid tablet from Anemarrhenae rhizoma. J Chromatogr B Anal Technol Biomed Life Sci. 2016;1026:134-44.

29. Hattori M, Shu YZ, Shimizu M, Hayashi T, Morita N, Kobashi K, et al. Metabolism of paeoniflorin and related compounds by human intestinal bacteria. Chem Pharm Bull. 1985;33:3838-46.
30. Shen L, Hillebrand A, Wang DQ, Liu M. Isolation and primary culture ofrat hepatic cells. J Vis Exp. 2012;64:3917.

31. Bi X, Gong M, Di L. Review on prescription compatibility of shaoyao gancao decoction and reflection on pharmacokinetic compatibility mechanism of traditional chinese medicine prescription based on in vivo drug interaction of main efficacious components. Evid Based Complement Altern Med. 2014;2014:208129.

32. Cong Y, Wang LM, Peng RJ, Zhao Y, Bai F, Yang C, et al. Timosaponin Alll induces antiplatelet and antithrombotic activity via Gq-mediated signaling by the thromboxane A2 receptor. Sci Rep. 2016;6:38757.

33. Fong S, King F, Zhao XY, Cohen I, Shtivelman E. Timosaponin A3 is a steroidal saponin from Anemarrhena asphodeloides that has a selective cytotoxic activity towards cancer cells. Cancer Res. 2009;69:615.

34. Lee B, Jung K, Kim DH. Timosaponin Alll, a saponin isolated from Anemar-rhena asphodeloides, ameliorates learning and memory deficits in mice. Pharmacol Biochem Behav. 2009;93:121-7.

35. Bissell DM, Gores GJ, Laskin DL, Hoofnagle JH. Drug-induced liver injury: mechanisms and test systems. Hepatology. 2001;33:1009-13.

36. Gold-Smith F, Fernandez A, Bishop K. Mangiferin and cancer: mechanisms of action. Nutrients. 2016;8:E396.

37. Saha S, Rashid K, Sadhukhan P, Agarwal N, Sil PC. Attenuative role of mangiferin in oxidative stress-mediated liver dysfunction in arsenic-intoxicated murines. Biofactors. 2016;42:515-32.

38. Pan C-W, Pan Z-Z, Hu J-J, Chen W-L, Zhou G-Y, Lin W, et al. Mangiferin alleviates lipopolysaccharide and d-galactosamine-induced acute liver injury by activating the Nrf2 pathway and inhibiting NLRP3 inflammasome activation. Eur J Pharmacol. 2016;770:85-91.

39. Han D, Chen C, Zhang C, Zhang Y, Tang X. Determination ofmangiferin in rat plasma by liquid-liquid extraction with UPLC-MS/MS. J Pharm Biomed Anal. 2010;51:260-3.

40. Hou C. Theoretical study of antioxidative ability and antioxidative mechanism of norathyriol in solution. Comput Theor Chem. 2014;1028:87-91. 